DOI: 10.17533/udea.efyd.v33n1a08

URL DOI: http://doi.org/10.17533/udea.efyd.v33n1a08

\title{
FACTORES ASOCIADOS CON LA ADHERENCIA A LA ACTIVIDAD FÍSICA EN EL TIEMPO LIBRE
}

\author{
FATORES ASSOCIADOS COM A ADERÊNCIA À ATIVIDADE \\ FÍSICA NO TEMPO LIBRE
}

\section{ASSOCIATED FACTORS WITH ADHERENCE TO PHYSICAL ACTIVITY IN LEISURE TIME}

\section{Elkin Fernando Arango Vélez}

Mag ster en Epidemiolog a Cl nica, Especialista en Medicina Deportiva.

Profesor e Investigador del Grupo de Investigaci n en Ciencias Aplicadas a la Actividad F sica y el Ejercicio (GRICAFDE) del Instituto de Educaci n F sica de la Universidad de Antioquia (Colombia)

elkinarango@yahoo.com

\section{Fredy Alonso Patiño Villada}

Doctorando en Ciencias de la Actividad F sica y el Deporte en la Universidad de Le n, Magister en Salud Publica por la Universidad de Antioquia.

Profesor e Investigador del Grupo de Investigaci n en Ciencias Aplicadas a la Actividad F sica y el Ejercicio (GRICAFDE) del Instituto de Educaci n F sica de la Universidad de Antioquia (Colombia)

fredy.patino@udea.edu.co

\section{Gildardo Díaz-Cardona}

Mag ster en Motricidad y Desarrollo Humano por la Universidad de Antioquia 
Profesor Instituto de Educaci n F sica, y Coordinador del programa PROSA de la Direcci n de Bienestar Universitario de la Universidad de Antioquia (Colombia).

coorprosa@gmail.com

\section{RESUMEN}

Introducci n: existe en Colombia poco conocimiento respecto a los factores asociados con la adherencia al ejercicio. Objetivo: evaluar los factores asociados con la adherencia a la actividad $f$ sica en el tiempo libre (AFTL) de los adultos que participan en un programa de actividad f sica (AF). M todos: se realiz un estudio prospectivo de Casos y Controles. Se defini como caso a quien realiz durante un periodo de 5 meses un promedio 150 minutos o m s de AFTL por semana a una intensidad moderada o 75 minutos a una intensidad vigorosa; como control se tom a quien no cumpli con los anteriores requisitos. Se evaluaron variables independientes sociodemogr ficas, factores de riesgo cardiovascular y osteomuscular, y las motivacionales para la pr ctica del ejercicio. Los datos se analizaron por medio de un modelo de regresi $\mathrm{n}$ log stica multivariado. Resultados: la muestra la conformaron 176 personas usuarias de un programa de AF, en su mayor a mujeres (69,3\%); el promedio de edad fue de 55,7 a os. Las variables asociadas con la adherencia a la AFTL fueron el antecedente personal de artrosis (OR 0,392; IC95\% 0,184-0,837; p=0,016) y la edad (OR 1,027; IC95\% 1,001-1,054; $p=0,041$ ), las cuales explicaron el 8,0\% de la variabilidad. Conclusiones: las variables que se asociaron en forma independiente con la adherencia a la AFTL fueron la edad y el antecedente personal de artrosis. Se requiere realizar nuevos estudios que incluyan otras variables que puedan explicar en forma $\mathrm{m}$ s global este fen meno.

PALABRAS CLAVE: Adherencia, actividad f sica, factores asociados, tiempo libre

\section{RESUMO}

Introduç o: Existe na Col mbia pouco conhecimento a respeito dos fatores associados com a ader ncia ao exerc cio. Objetivo: Avaliar os fatores associados com a ader ncia atividade $f$ sica no tempo livre (AFTL) dos adultos que participam em um programa de atividade $f$ sica (AF). $M$ todos: Se realizou um estudo prospectivo de casos e controles. Definiu-se como caso a quem realizou durante um per odo de 5 meses em $\mathrm{m}$ dia 150 minutos ou mais de AFTL por semana, a uma intensidade moderada ou 75 minutos a uma intensidade vigorosa; como controle tomou-se a quem $\mathrm{n} o$ cumpriu com os requisitos anteriores. Avaliou-se vari veis independentes 
s cios demogr ficas, fatores de risco cardiovascular, osteomuscular e as motivaç es para a pr tica de exerc cios. Analisaram-se os dados por meio de um modelo de regress o log stica multivariado. Resultados: conformaram a amostra 176 pessoas usu rias de um programa de AF, em sua maioria mulheres (69,3\%); a $\mathrm{m}$ dia de idade foi de 55,7 anos. As vari veis associadas com a ader ncia a AFTL foram o antecedente pessoal de artrose (OR 0,392; IC95\% 0,184-0,837; $p=0,016)$ e a idade (OR 1,027; IC95\% 1,001-1,054; $p=0,041)$, os quais explicaram os $8 \%$ de variabilidade. Conclus es: As vari veis que se associaram de forma independente com a ader ncia AFTL foram a idade e o antecedente pessoal de artrose. Faz-se necess rio realizar novos estudos que incluam outras vari veis que possam explicar de forma mais global este fen meno.

PALAVRAS-CHAVE: Ader ncia, atividade $\mathrm{f}$ sica, fatores associados, tempo livre

\section{ABSTRACT}

Introduction: In Colombia there is a low knowledge related to the associated factors with adherence to exercise. Objective: To evaluate the associated factors with adherence to the physical activity in leisure time in adults (PALT) who participate in a physical activity program. Methods: The study was a prospective case-control. A case was defined as who performed during five months an average of 150 minutes or more per week of PALT, with a moderate intensity or strong intensity of 75 minutes or more of PALT per week; as a control was had in consideration, people who did not meet the above criteria. The independent variables measured were: sociodemographic factors, cardiovascular, musculoskeletal risk factors, and the motivation to exercise. The data was analyzed through a varied regressive logistic model. Results: The sample involved 176 female users of a PA program (69.3\%); with an average age of 55.7 years old. The associated variables with the PALT adherence were the personal osteoarthritis record (OR 0,392; IC95\% 0,184 - 0,837; $\mathrm{P}=0,016)$ and the age (OR 1,027; IC95\% 1,001-1,054; $p=0,041)$, which explained $8,0 \%$ of the variability. Conclusions: The variables that were independently associated to the adherence to PALT were age, and the personal osteoarthritis record. There is a necessity of new studies that include new variables to allow a global analysis of the phenomena.

KEY WORDS: Adherence, physical activity, associated factors, leisure time, adherence 


\section{INTRODUCCIÓN}

La actividad $f$ sica en el tiempo libre (AFTL) se reconoce como una herramienta til para la prevenci $\mathrm{n}$ y tratamiento de las enfermedades $\mathrm{cr}$ nicas no transmisibles (ECNT) como la hipertensi $\mathrm{n}$ arterial, la diabetes mellitus, las dislipidemias, las enfermedades cardiovasculares, la osteoporosis, la artrosis, el asma y la enfermedad pulmonar obstructiva $\mathrm{cr}$ nica, as como para la mejora de la calidad de vida relacionada con la salud (CVRS). Los beneficios con la pr ctica de actividad $f$ sica se logran mediante una buena adherencia, cumpliendo de manera adecuada con el plan de ejercicio recomendado (Burke, Dunbar-Jacob \& Hill, 1997; Woodard \& Berry, 2001). A pesar de lo anterior, la adherencia a las recomendaciones de actividad $\mathrm{f}$ sica (AF) es baja, lo que se evidencia en que hasta un $50 \%$ de las personas que inician un programa de AF se retiran del mismo durante los primeros 6 meses, tasas de deserci n que en los adultos mayores son incluso m s altas (Findorff, Wyman \& Gross, 2009).

Se han hecho diversas investigaciones con el fin de conocer los factores que se asocian con la adherencia a las recomendaciones de $\mathrm{AF}$, con resultados que distan de ser un nimes. Es as como en aspectos sociodemogr ficos como el sexo, estudios realizados en Suecia (Bergman, Grjibovski, Hagstromer, Bauman \& Sjostrom, 2008) y Madrid (Meseguer, Gal n, Herruzo, ZorriIla, \& Rodr guez-Artalejo, 2009) muestran una mayor adherencia en los hombres, mientras que otros autores no encontraron diferencias significativas entre los sexos en la adherencia a la AF general (Leijon et al., 2010) y al yoga en particular (Flegal, Kishiyama, Zajdel, Haas \& Oken, 2007), con seguimientos que variaron entre 3 y 12 meses, donde el estado de nimo y el apoyo social tuvieron mayor asociaci $\mathrm{n}$ con la adherencia que factores como el sexo. En lo referente a la edad existen datos contradictorios; mientras en un estudio se report mayor adherencia a la AFTL entre los 18-34 a os (Bergman et al., 2008), otro report una 
asociaci $\mathrm{n}$ inversa entre la edad y la adherencia (Meseguer et al., 2009), y uno $\mathrm{m}$ s observ mayor adherencia en las personas de mayor edad (Leijon et al., 2010). Otras caracter sticas, como tener un nivel educativo alto, estar soltero (especialmente en las mujeres) y tener una actividad laboral de baja intensidad, parecen asociarse con una mayor adherencia a las recomendaciones de AFTL (Bergman et al., 2008; Meseguer et al., 2009).

As mismo, aunque la evidencia cient fica apoya el beneficio de la AF en personas con enfermedades cr nicas, la adherencia de los pacientes a esta pr ctica oscila entre el 20-80\% (Cupples \& McKnight, 1994; Kravitz et al., 1993; Robertson \& Keller, 1992). En forma m s espec fica, quienes padecen enfermedades cardiovasculares, a pesar de conocer las mejor as a corto plazo en los resultados cl nicos, la mitad de quienes participan en programas de rehabilitaci $\mathrm{n}$ card aca los abandonaron durante el primer a o (Ades, Waldmann, McCann, \& Weaver, 1992; Oldridge, 1984; Oldridge \& Streiner, 1990).

De otro lado, padecer sobrepeso y obesidad disminuye la probabilidad de adherirse a las recomendaciones de AFTL, en comparaci n con personas normopeso (Meseguer et al., 2009; Perri, McAdoo, McAllister, Lauer \& Yancey, 1986); y participar en programas de ejercicio con supervisi $n$ en el hogar mejora la adherencia a los mismos, lo cual se evidencia en personas que padecen enfermedad pulmonar obstructiva cr nica (EPOC) (Carrieri-Kohlman et al., 2005; Grosbois et al., 1999; Steele et al., 2008).

Otro aspecto a tener en cuenta en la adherencia a la AFTL es la motivaci $\mathrm{n}$ de las personas para realizar dichas actividades; as, en personas de edad avanzada las motivaciones son I dicas, relajantes y de relaci $n$, concediendo menor importancia a aspectos como la imagen corporal y la est tica; en la poblaci $n$ general se reporta que los factores motivacionales $\mathrm{m} \mathrm{s}$ importantes para la pr ctica de AFTL son el fitness-salud, seguido por el disfrute y la apariencia f sica (Hell n, Moreno \& Rodr guez, 2004; Ryan, Frederick, Lepes, Rubio \& Sheldon, 1997; Sit, Kerrb \& Wong, 2008). 
En Colombia, y particularmente en Medell n, se carece de estudios suficientes que permitan conocer el grado de adherencia a las recomendaciones de AFTL necesarias para lograr las adaptaciones fisiol gicas y mentales que permitan una disminuci $\mathrm{n}$ de la prevalencia e incidencia de las ECNT previamente mencionadas, con el fin de reducir el impacto delet reo de estas patolog as sobre la CVRS y la reducci $\mathrm{n}$ de la expectativa de vida de las personas. Adem s, se tienen falencias en el conocimiento sobre los factores asociados a la adherencia a la AFTL. Por dichas razones este estudio tuvo como objetivo determinar los factores asociados con la adherencia a la AFTL de un grupo de adultos que participan en un programa de AF dirigida.

\section{METODOLOGÍA}

Se realiz un estudio prospectivo de casos y controles. Se estudiaron personas pertenecientes al Programa de Salud Psicof sica (PROSA) de la Universidad de Antioquia durante el a o 2011. Para el c Iculo del tama o de la muestra se consideraron: OR de 3, error alfa del $5 \%$, potencia del $80 \%$, proporci n de exposici $n$ en los casos del $25 \%$ y proporci $n$ de exposici $n$ en los controles del $10 \%$. Se determin una raz $\mathrm{n}$ de un caso por cada dos controles, con lo que se obtuvo un tama o de la muestra de 214 participantes, de las cuales 72 deb an ser casos y 142 controles. Es de anotar que la muestra final la conformaron 176 personas, que corresponden al 82,2\% del tama o de la muestra que se calcul para el estudio.

Se definieron como casos (adheridos) las personas que durante los Itimos cinco meses realizaron en promedio 150 minutos o $\mathrm{m}$ s de AFTL por semana, a una intensidad moderada; o $75 \mathrm{mi}-$ nutos o $\mathrm{m}$ s de AFTL por semana, a una intensidad vigorosa. Los controles (no adheridos) fueron aquellas personas que durante el mismo periodo de tiempo realizaron un promedio inferior a 
150 minutos de AFTL por semana, a una intensidad moderada; o menor a 75 minutos de AFTL por semana, a una intensidad vigorosa.

Se incluyeron personas pertenecientes al programa PROSA al inicio de 2011. Fueron excluidas las personas que no tuvieron al menos una evaluaci $n$ por medicina del deporte durante 2010 y los primeros 3 meses de 2011, y quienes no firmaron el consentimiento informado.

Se hizo control de los sesgos de informaci $n$ al capacitar a los encuestadores en el manejo de los instrumentos de recolecci $n$ de los datos, con el fin de que se procesaran en forma estandarizada en cada una de sus etapas. Adem s, se llev a cabo un control de calidad de los formatos diligenciados durante el trabajo de campo y se entreg un "Manual del encuestador", que permiti resolver dudas sobre la forma de tomar los datos. A los participantes se les explicaron los objetivos del estudio y cada uno de los procedimientos que se realizaron dentro de I. Se garantiz el manejo confidencial de los datos, los cuales solo se utilizar an para fines acad micos y de la investigaci $n$. Se realiz una prueba piloto con el fin de conocer las dificultades durante la aplicaci $n$ de la encuesta y la evaluaci $n$ de campo. Los datos de fuente secundaria se tomaron de la historia $\mathrm{cl}$ nica $\mathrm{m}$ dico-deportiva de cada participante, la cual fue dise ada con el fin de obtener informaci $n$ como la requerida para esta investigaci n. Los sesgos de selecci $n$ se controlaron con la verificaci $\mathrm{n}$ de la pertenencia de cada usuario al programa previo al ingreso al estudio y con la evaluaci $n$ en forma estricta de la adherencia a la AFTL en forma individual.

La variable dependiente (adherencia a AFTL) se obtuvo en forma prospectiva con seguimiento individual semanal a cada persona; las independientes sociodemogr ficas (sexo, edad, nivel educativo, estrato socioecon mico, ocupaci n y estado civil) y cl nicas (hipertensi $\mathrm{n}$ arterial, diabetes mellitus, dislipidemias, tabaquismo, consumo de licor, artrosis, dolor osteomuscular $\mathrm{cr}$ nico, enfermedad cardiovascular, consumo de medicamentos, 
antecedente familiar de enfermedad cerebrovascular prematura, obesidad por ndice de Masa Corporal (IMC), obesidad central y obesidad por porcentaje de grasa), se obtuvieron de fuente secundaria de las historias $\mathrm{m}$ dico-deportivas de cada participante; las variables motivacionales (disfrute, apariencia, social, fitness y competencia) se midieron por medio de un cuestionario estructurado, a partir de la escala MPAM-R (Motives for Physical Measure-Revised) (Ryan et al., 1997), que mide los motivos de pr ctica de AF a trav s de 30 tems mediante una escala tipo Likert de siete puntos, donde 1 corresponde a "nada verdadero para mi" y 7 a "totalmente verdadero para mi"; estos tems se agrupan en 5 factores denominados: "disfrute" (realizo AF porque es divertido), "apariencia" (realizo AF porque quiero mantener mi peso para tener buena imagen), "social" (realizo AF porque quiero conocer a nuevas personas), "fitness" (realizo AF porque quiero mejorar mi aptitud cardiovascular), "competencia" (realizo AF porque me gustan los desaf os); esta escala se valid en Espa a, donde se reportaron valores del alfa de Cronbach de 0,92; 0,91; 0,$83 ; 0,78$ y 0,88 , respectivamente para cada uno de los factores (Moreno, Cervell \& Mart nez 2007).

Definici $n$ de las variables cl nicas: hipertensi $\mathrm{n}$ arterial (HTA) a quienes tuvieron el antecedente de la condici $n$, o que consu$\mathrm{m}$ an alg $\mathrm{n}$ medicamento antihipertensivo, o reporte en la historia $\mathrm{cl}$ nica de una presi $\mathrm{n}$ arterial sist lica $\geq 140 \mathrm{mmHg}$ y/o presi $\mathrm{n}$ arterial diast lica $\geq 90 \mathrm{mmHg}$ (Chobanian et al., 2003); diabetes mellitus (DM) a personas con el antecedente personal de esta condici n, o que consum an hipoglicemiantes orales o se aplicaran insulina (American Diabetes Association, 2009); se consideraron con dislipidemias a personas con historia de estos trastornos, o que consum an alg $\mathrm{n}$ medicamento hipolipemiante (Expert Panel on Detection, Evaluation, and Treatment of High Blood Cholesterol in Adults, 2001); obesidad a personas con un IMC $\geq 30 \mathrm{~kg} / \mathrm{m}^{2}$, con sobrepeso un IMC $\geq 25 \mathrm{~kg} / \mathrm{m}^{2}$ y menor de $30 \mathrm{~kg} / \mathrm{m}^{2}$ (Eckel, 2008); obesidad central $(\mathrm{OC})$, hombres con un per metro abdominal 
$\geq 90 \mathrm{~cm}$ y mujeres con valores $\geq 80 \mathrm{~cm}$ (Alberti et al., 2009); tabaquismo personas que fumaron cigarrillo al menos una vez durante el Itimo a o (Lanas et al., 2007); consumidores altos de licor fueron quienes reconocieron un consumo de alcohol superior al percentil 50 del consumo en gramos por semana; el antecedente familiar de ECV prematura se defini como la presencia actual o antigua en un familiar de primer grado de consanguinidad de enfermedad coronaria, enfermedad cerebro vascular, enfermedad arterial perif rica oclusiva o muerte s bita; osteoartrosis quienes tuvieron el antecedente personal en la historia cl nica; y dolor osteomuscular $\mathrm{cr}$ nico (DOC) quienes presentaron dicho reporte en la historia cl nica, sin importar el origen.

\section{Evaluación del desenlace}

Adherencia a la actividad $f$ sica en el tiempo libre (AFTL): en forma semanal, durante un periodo de 16 semanas, se indag con cada participante sobre la pr ctica de AFTL durante los $7 \mathrm{~d}$ as previos. Se registr el $\mathrm{n}$ mero de sesiones por semana, tiempo promedio en cada sesi n, intensidad de trabajo y el tipo de AF que realiz . Al finalizar el periodo de observaci $n$, el gasto cal rico total de las actividades de resistencia aer bica fueron calculadas teniendo en cuenta el tipo de ejercicio, el peso del individuo y el tiempo total de la sesi n (Williams, 2002). Para calcular el gasto energ tico durante el ejercicio de fuerza, cuya caracter stica fue el entrenamiento en circuito, se emplearon las $f$ rmulas de Wilmora et al., (para hombres gasto energ tico $=0,116 \mathrm{cal} / \mathrm{kg} / \mathrm{min}$; para las mujeres gasto energ tico $=0,100 \mathrm{cal} / \mathrm{kg} / \mathrm{min})(\mathrm{S}$ nchez, 2004); el resultado se calcul en calor as y con este dato se estimaron los MET que cada individuo gast por semana.

\section{Análisis estadístico}

Las variables cualitativas se resumieron con frecuencias absolutas y relativas. En las variables cuantitativas se verific el supuesto de normalidad por medio de la prueba de Kolmogorov Smirnov (KS); 
aquellas con distribuci $\mathrm{n}$ normal se resumieron con medias y desviaci $n$ est ndar (DE); las que mostraron distribuci n no normal, se resumieron con medianas y rangos intercuart licos (RI). La asociaci $n$ cruda entre las variables dicot micas y el desenlace de inter $\mathrm{s}$, se realiz mediante la prueba de $\mathrm{Chi}^{2}$. Se implement un modelo de regresi $\mathrm{n}$ log stica binaria multivariado, con el prop sito de obtener un modelo explicativo con las variables asociadas de forma independiente y significativa con la posibilidad de cumplir las pautas de AF para la salud; se incluyeron en el modelo las variables que en el an lisis bi-variado cumplieron el criterio de Hosmer-Lemeshow de un valor de $p<0,25$. Adem $s$, se calcul el coeficiente de determinaci $n\left(R^{2}\right)$ de Nagelkerke, para indicar el porcentaje de la varianza explicado por las variables incluidas en el modelo de regresi $\mathrm{n}$ log stica binaria. Todos los an lisis se realizaron con un nivel de significancia $p<0,05$. Se utiliz el paquete estad stico SPSS versi n 19 (Chicago, IL).

\section{Aspectos éticos}

Se garantiz la protecci $\mathrm{n}$ de la intimidad de las personas de acuerdo a la Declaraci n de Helsinki de 2008 (Asociaci n Medica Mundial, 2008) y las disposiciones de la resoluci n 8430 de 1993 del Ministerio de la Salud de la Rep blica de Colombia (Ministerio de Salud, 1993). Todas las personas estudiadas fueron informadas sobre los objetivos del estudio y la utilizaci $n$ exclusiva de los datos para fines cient ficos y de planeaci $n$ del programa para la salud psico-f sica (PROSA). Todos los participantes firmaron el consentimiento informado.

\section{RESULTADOS}

\section{Aspectos sociodemográficos}

Conformaron la muestra 176 personas usuarias de un programa de AF dirigido, con un promedio de edad de 55,7 a os (DE 
$13,1)$; el $69,3 \%$ fueron mujeres y los a os de educaci $n$ formal alcanzaron una media de 15,3 (DE 5,7). Una de cada dos personas $(48,9 \%)$ tuvieron uni $n$ marital de hecho y el $7,4 \%$ hab an enviudado; las ocupaciones $\mathrm{m} s$ prevalentes fueron el ser empleado $(35,8 \%)$ y estar jubilado $(31,8 \%)$, y la menos frecuente fue el trabajo familiar sin remuneraci $\mathrm{n}$ (TFSR); 4 de cada 10 personas tuvieron ingresos menores a tres salarios $\mathrm{m}$ nimos mensuales (Ver tablas 1 y 3 ).

\begin{tabular}{|c|c|c|c|}
\hline & & $\mathbf{n}$ & $\%$ \\
\hline \multirow[t]{2}{*}{ Sexo } & Mujer & 122 & 69,3 \\
\hline & Hombre & 54 & 30,7 \\
\hline \multirow[t]{4}{*}{ Estado civil } & Soltero & 58 & 33,0 \\
\hline & Casado / Unión libre & 86 & 48,9 \\
\hline & Viudo & 13 & 7,4 \\
\hline & Separado /divorciado & 19 & 10,8 \\
\hline \multirow[t]{5}{*}{ Ocupación } & TFSR* & 21 & 11,9 \\
\hline & Empleado & 63 & 35,8 \\
\hline & Jubilado & 56 & 31,8 \\
\hline & Docente & 33 & 18,8 \\
\hline & Otro & 3 & 1,7 \\
\hline \multirow{3}{*}{$\begin{array}{l}\text { Ingresos } \\
\text { en salarios } \\
\text { mínimos }\end{array}$} & 0 a 2 & 73 & 41,5 \\
\hline & 3 a 4 & 49 & 27,8 \\
\hline & $\geq 5$ & 54 & 30,7 \\
\hline
\end{tabular}

*Trabajo familiar sin remuneración

Tabla 1. Características sociodemográficas, PROSA -2011 (n=176) 
Prevalencia de la adherencia a la AFTL y de algunas ECNT La adherencia a la AFTL fue del $63,1 \%$. Las ECNT m s prevalentes fueron las dislipidemias (50,6\%), el DOC $(50,6 \%)$ y la obesidad, tanto por porcentaje de grasa como por grasa visceral. Se encontr una baja frecuencia de fumadores activos $(6,8 \%)$ y una alta prevalencia de personas que lograron dejar de fumar $(17,6 \%)$ (Ver tabla 2$)$.

\begin{tabular}{l|c|c}
\hline & $\mathbf{n}$ & $\%$ \\
\hline Adherencia a la AF & 111 & 63,1 \\
HTA & 47 & 26,7 \\
DM & 7 & 4,0 \\
Dislipidemia & 89 & 50,6 \\
Fumador & 12 & 6,8 \\
Exfumador & 31 & 17,6 \\
Nunca ha fumado & 133 & 75,6 \\
AFEC* & 45 & 25,6 \\
Artrosis & 39 & 22,2 \\
DOC** & 89 & 50,6 \\
Obesidad IMC & 19 & 10,8 \\
Obesidad central & 63 & 35,8 \\
Obesidad (\% grasa) & 69 & 39,2 \\
\hline
\end{tabular}

*Antecedentes familiares de enfermedad cardiovascular; **dolor osteomuscular crónico

Tabla 2. Prevalencia de la Adherencia a la Actividad Física y de algunas ECNT, PROSA - $2011(n=176)$

Composición corporal y aspectos motivacionales

Los valores de las variables antropom tricas se pueden consultar en la tabla 3. En los aspectos motivacionales para la pr ctica de AFTL, la dimensi n "fitness" mostr el puntaje promedio m s 
alto $(6,6 ; \mathrm{DE} 0,6)$ y la "apariencia" arroj el valor m s bajo $(5,1$; DE 1,5$)$ (Ver tabla 3).

\begin{tabular}{l|c|c|c|c|c}
\hline & Media & DE & Mediana & P 25 & P 75 \\
\hline Edad (años) & 55,7 & 13,1 & 57,0 & 46,0 & 65,0 \\
Años de estudio & 15,3 & 5,7 & 16,0 & 11,0 & 19,0 \\
IMC (kg/m²) & 25,0 & 3,5 & 24,8 & 22,2 & 27,5 \\
Circunferencia de & 80,1 & 10,6 & 79,0 & 71,5 & 88,0 \\
cintura (cm) & 33,1 & 6,5 & 33,4 & 28,4 & 38,1 \\
\% grasa & 18,0 & 36,7 & 0,0 & 0,0 & 30,0 \\
$\begin{array}{l}\text { Consumo de licor } \\
\text { (gr/sem) }\end{array}$ & 6,0 & 0,9 & 6,1 & 5,5 & 6,7 \\
Disfrute* & 5,1 & 1,5 & 5,3 & 4,3 & 6,2 \\
Apariencia* & 5,4 & 1,5 & 5,8 & 4,8 & 6,8 \\
Social* & 6,6 & 0,6 & 6,8 & 6,4 & 7,0 \\
Fitness* & 5,3 & 1,3 & 5,5 & 4,7 & 6,3 \\
Competencia* & & & & \\
\hline
\end{tabular}

gr/sem: gramos por semana; *puntajes de los aspectos motivacionales para realizar AF; DE: desviación estándar; P. percentil

Tabla 3. Composición Corporal y Aspectos Motivacionales.

PROSA - $2011(n=176)$

Adherencia a la AFTL de acuerdo a los aspectos sociodemográficos

Al comparar la adherencia a la AFTL de acuerdo a las variables sociodemogr ficas, no se encontraron diferencias estad sticamente significativas en ninguna de ellas. Se observ que las personas viudas y quienes reportaron ingresos mensuales de $\mathrm{m} \mathrm{s}$ de $5 \mathrm{sa}-$ larios $\mathrm{m}$ nimos estuvieron $\mathrm{m} \mathrm{s}$ adheridos a la AFTL (Ver tabla 4). 


\begin{tabular}{|c|c|c|c|}
\hline & & $\mathbf{n}$ & $\%$ \\
\hline \multirow[t]{2}{*}{ Sexo } & Mujer & 76 & 62,3 \\
\hline & Hombre & 35 & 64,8 \\
\hline \multirow[t]{3}{*}{ Edad } & tertil1 & 35 & 57,4 \\
\hline & tertil2 & 36 & 65,5 \\
\hline & tertil3 & 40 & 66,7 \\
\hline \multirow[t]{3}{*}{ Años estudio } & tertil1 & 44 & 64,7 \\
\hline & tertil2 & 34 & 61,8 \\
\hline & tertil3 & 33 & 62,3 \\
\hline \multirow[t]{4}{*}{ Estado civil } & Soltero & 36 & 62,1 \\
\hline & Casado / Unión libre & 54 & 62,8 \\
\hline & Viudo & 9 & 69,2 \\
\hline & Separado /divorciado & 12 & 63,2 \\
\hline \multirow[t]{5}{*}{ Ocupación } & TFSR & 13 & 61,9 \\
\hline & Empleado & 37 & 58,7 \\
\hline & Jubilado & 38 & 67,9 \\
\hline & Docente & 20 & 60,6 \\
\hline & Otro & 3 & 100,0 \\
\hline \multirow[t]{3}{*}{ Ingresos* } & 0 a 2 & 44 & 60,3 \\
\hline & 3 a 4 & 29 & 59,2 \\
\hline & $\geq 5$ & 38 & 70,4 \\
\hline
\end{tabular}

TFSR: trabajo familiar sin remuneración; *en salarios mínimos mensuales

Tabla 4. Adherencia a la AF según aspectos sociodemográficos.

PROSA - $2011(n=176)$ 


\section{Adherencia a la AFTL según algunas ECNT}

En las personas hipertensas, diab ticas, dislipid micas, fumadoras y obesas se encontr una menor proporci $\mathrm{n}$ de adherencia a las recomendaciones de $\mathrm{AF}$ con respecto a quienes no presentaron estas condiciones patol gicas, ninguna de ellas con diferencias estad sticamente significativas. Se destaca, que el ser fumador redujo la frecuencia de adherencia al compararlo con los exfumadores $(41,7 \%$ vs $61,3 \% ; p>0,05)$, diferencia que, aunque no fue significativa desde el punto de vista estad stico, s lo es para la pr ctica. Las personas con alg $\mathrm{n}$ tipo de artrosis mostraron una menor adherencia a la AFTL $(48,7 \%$ vs. $67,2 \%$; $p=0,035)$, tendencia que tambi $\mathrm{n}$ se encontr en quienes refirieron tener DOC (Ver tabla 5).

\begin{tabular}{l|c|c|c}
\hline & & $\mathbf{n}$ & $\%$ \\
\hline HTA & NO & 83 & 64,3 \\
DM & SI & 28 & 59,6 \\
Dislipidemia & NO & 107 & 63,3 \\
& SI & 4 & 57,1 \\
Tabaquismo & NO & 55 & 63,2 \\
& SI & 56 & 62,9 \\
& Fumador & 5 & 41,7 \\
AFEC** & Exfumador & 19 & 61,3 \\
& Nunca ha fumado & 87 & 65,4 \\
Artrosis & NO & 84 & 64,1 \\
& SI & 27 & 60,0 \\
DOC*** & NO & 92 & $67,2 *$ \\
& SI & 19 & 48,7 \\
Obesidad IMC & NO & 58 & 66,7 \\
& SI & 53 & 59,6 \\
& NO & 100 & 63,7 \\
& & 11 & 57,9 \\
\hline
\end{tabular}




\begin{tabular}{l|c|c|c}
\hline & & $\mathbf{n}$ & $\%$ \\
\hline Obesidad central & NO & 74 & 65,5 \\
& SI & 37 & 58,7 \\
Obesidad (\% grasa) & NO & 68 & 63,6 \\
& SI & 43 & 62,3 \\
\hline
\end{tabular}

*p<0,05; **antecedente familiar de enfermedad cardiovascular; $* * *$ dolor osteomuscular crónico

Tabla 5. Adherencia a la AFTL según algunas ECNT. PROSA, $2011(n=176)$

Adherencia a la AFTL de acuerdo a los aspectos motivacionales

Las medianas de los puntajes de las diferentes dimensiones motivacionales no mostraron diferencias estad sticamente significativas entre las personas adheridas y no adheridas a la AFTL. Los puntajes $\mathrm{m}$ s altos se encontraron en las dimensiones "Fitness" y "Disfrute" (Ver tabla 6).

\begin{tabular}{l|c|c|c|c}
\hline \multirow{2}{*}{} & \multicolumn{2}{|c|}{ No adherido } & \multicolumn{2}{c}{ Adherido } \\
\cline { 2 - 5 } & Mediana & RI & Mediana & RI \\
\hline Disfrute & 6,1 & $5,4-6,7$ & 6,1 & $5,5-6,7$ \\
Apariencia & 5,3 & $4,3-6,3$ & 5,3 & $4,1-6,1$ \\
Social & 5,5 & $4,1-6,6$ & 5,7 & $5,0-6,7$ \\
Fitness & 6,8 & $6,2-7,0$ & 6,8 & $6,4-7,0$ \\
Competencia & 5,5 & $4,7-6,4$ & 5,5 & $4,5-6,3$ \\
\hline
\end{tabular}

Tabla 6. Adherencia a la AF según Aspectos Motivacionales. PROSA, $2011(n=176)$

Variables asociadas a la AFTL

En el an lisis de regresi $\mathrm{n}$ log stica multivariado, se encontr que las variables asociadas con la adherencia a la AFTL fueron la 
edad (OR 1,027; IC95\% 1,001-1,054; $p=0,041)$ y el antecedente personal de alg $\mathrm{n}$ tipo de artrosis (OR 0,392; IC95\% 0,184$0,837 ; p=0,016)$; se debe tener en cuenta que en este modelo se incluy el antecedente de tabaquismo, debido a que al retirarlo del mismo se confunde el valor del OR de la variable edad; estas tres variables explicaron el $8,0 \%\left(R^{2}=0,08\right)$ de la variabilidad de la adherencia a la AFTL.

\section{DISCUSIÓN}

El principal hallazgo de este estudio fue que las variables que se asociaron en forma independiente a la adherencia a las recomendaciones de AFTL para la salud fueron el tener una mayor edad, la cual se asoci en forma directa, y el tener diagn stico de osteoartrosis degenerativa, cuya asociaci $\mathrm{n}$ fue inversa.

La adherencia a las recomendaciones de AFTL en este estudio fue del $63,1 \%$, lo que supera las proporciones que se reportan en otras poblaciones locales y nacionales (Arango, Pati o, Quintero \& Arenas, 2011; Mantilla, 2006; Rodr guez et al., 2009) y se asemeja a la que reportaron en un estudio poblacional en Suecia, cuyo valor fue del $63 \%$ (Bergman et al., 2008). A este respecto se debe tener en cuenta que la adherencia a la AF regular, principalmente de tipo aer bico, se asocia a menor riesgo de mortalidad por todas las causas, con un mayor beneficio en las personas que tienen al menos una ECNT y son mayores de 65 a os (Schoenborn \& Stommel, 2011).

En lo referente a la edad, la asociaci $n$ directa que se hall en este estudio con el cumplimiento de las recomendaciones de AFTL, contrasta con reportes donde la relaci $n$ fue inversa (Bergman et al., 2008; Meseguer et al., 2009), lo que se pudiese explicar por una mayor conciencia de la importancia de la pr ctica de AFTL para mantener y mejorar las condiciones de salud a medida que se incrementa la edad; este hallazgo lleva a buscar las causas 
por las cuales las personas $\mathrm{m} \mathrm{s}$ venes tienen menor adherencia a la AF, con el fin de minimizar dicho fen meno y, por ende, reducir el impacto que pueden tener en ellas el desarrollo de las ECNT. Las dem $\mathrm{s}$ variables sociodemogr ficas no mostraron asociaci $\mathrm{n}$ independiente con la adherencia a la $\mathrm{AF}$, mientras que en otros estudios se reporta mayor adherencia en los hombres, en las personas con un mayor nivel educativo, solteras y con ingresos econ micos altos (Bergman et al., 2008; Meseguer et al., 2009; Netz, Goldsmith, Shimony, Ben-Moshe \& Zeev, 2011).

En las personas con obesidad, tanto central como por IMC, se hall una menor proporci $\mathrm{n}$ de adherencia a la $A F$, valores que, aunque no fueron estad sticamente significativos, muestran una tendencia que se debe tener en cuenta por quienes ofrecen programas grupales de ejercicio $f$ sico, debido que la obesidad se comporta como causa y efecto de los bajos niveles de AF (Bergman et al., 2008). En este estudio se encontr asociaci $n$ inversa entre el antecedente de osteoartrosis y la adherencia a la AFTL; las dem s ECNT como DM, HTA, dislipidemia, DOC y tabaquismo, no se asociaron con dicho desenlace. En lo referente a la osteoartrosis, la baja adherencia de las personas con esta condici $n$ se puede deber a las limitaciones funcionales para realizar AF y al incremento de los s ntomas como el dolor que se puede dar en ellas; de otro lado, las personas con esta enfermedad tienen la creencia de que no deben realizar ejercicio por el posible da o que se puede generar en sus articulaciones; adem s, generalmente tienen una percepci $\mathrm{n}$ de deterioro sobre su estado de salud (Marks, 2012). El anterior hallazgo contrasta con el reporte de un estudio que se realiz en mujeres adultas mayores, donde no encontraron asociaci $n$ entre artrosis y la adherencia a un plan de caminata en la comunidad (Findorff et al., 2009).

Respecto a las motivaciones para la pr ctica de la AFTL, los puntajes $\mathrm{m} \mathrm{s}$ altos fueron para las dimensiones de "Fitness/salud" y "Disfrute", resultados que fueron similares a otro estudio con la adherencia a la actividad f sica en el tiempo libre 
donde estas dos dimensiones estuvieron entre las razones $\mathrm{m} \mathrm{s}$ importantes para la realizaci n de la AFTL (Aaltonen et al., 2012). En el an lisis de las motivaciones y la adherencia a la AFTL no se encontraron diferencias significativas; estas, al parecer, no se comportaron como un asunto relevante al momento de diferenciar las personas adheridas o no, contrario a un estudio transversal con poblaci $\mathrm{n}$ finlandesa donde los motivos relacionados con la destreza, la buena salud, los aspectos sociales, el estado psicol gico, el disfrute y la apariencia fueron significativamente m s importantes para las personas que cumpl an con las recomendaciones de $\mathrm{AF}$ (>5,3 METs/h/d a) comparadas con aquellas que estaban por debajo de los niveles necesarios (<2,3 METs h/ d a) (Aaltonen, Rottensteiner, Kaprio \& Kujala, 2013). Por otra parte, en un estudio con poblaci $n$ brasilera se encontr que las motivaciones para la realizaci $\mathrm{n}$ de AF estaban relacionadas con el tipo de actividad, la edad de las personas y el nivel educativo (de Andrade Bastos, Salguero, Gonzalez-Boto \& M rquez, 2006); aunque dichas asociaciones no fueron tratadas en este estudio, son aspectos que se deben considerar al momento de la planificaci $\mathrm{n}$ de los programas de AFTL, con lo cual se podr a alcanzar una mayor adherencia a los mismos.

Esta investigaci $\mathrm{n}$ tuvo algunas limitaciones a tener en cuenta en el momento de interpretar los resultados: 1) El tama o de la muestra y la relaci $n$ de casos y controles no fueron los que se esperaban, lo que afect la potencia del estudio; esto se explica por la falta de cumplimiento de los criterios de selecci n y falta de participaci $\mathrm{n}$ de algunas personas pertenecientes al programa PROSA; 2) El instrumento para valorar las motivaciones para la pr ctica de AF no cuenta con validaci $n$ en el contexto colombiano, lo que puede sesgar los resultados; 3) Parte de los datos de la pr ctica de AFTL se obtuvieron por autorreporte, lo que tiene impl cito el riesgo de sesgo de memoria.

En conclusi n, de los diferentes factores estudiados, se encontr que el tener antecedente personal de osteoartrosis y una 
mayor edad se asoci de forma independiente a la adherencia a la AFTL, factores que explicaron el $8,0 \%$ de la variabilidad. Este es el primer estudio del que se tiene conocimiento en Colombia, con el fin de determinar factores asociados a la adherencia a las recomendaciones mundiales de AF para la salud, en personas que participan de un programa de AF dirigida. Para futuras investigaciones, se sugiere tener en cuenta otros factores que pueden influir sobre la adherencia a la AF, como son la percepci $n$ del estado de salud, trastornos del estado de nimo como la ansiedad y la depresi n, el apoyo familiar y social, el consumo de medicamentos y las barreras ambientales.

AGRADECIMIENTOS: a los usuarios, monitores y estudiantes practicantes del Programa para la Salud Psicof sica PROSA de la Universidad de Antioquia, por su participaci n y colaboraci $n$ en el desarrollo de la investigaci $n$.

\section{REFERENCIAS}

1. Aaltonen, S., Leskinen, T., Morris, T., Alen, M., Kaprio, J., Liukkonen, J., \& Kujala, U. (2012). Motives for and barriers to physical activity in twin pairs discordant for leisure time physical activity for 30 years. Int J Sports Med, 33(2), 157-163.

2. Aaltonen, S., Rottensteiner, M., Kaprio, J., \& Kujala, U. M. (2013). Motives for physical activity among active and inactive persons in their mid-30s. Scand J Med Sci Sports,

3. Ades, P. A., Waldmann, M. L., McCann, W. J., \& Weaver, S. O. (1992). Predictors of cardiac rehabilitation participation in older coronary patients. Arch Intern Med, 152(5), 1033-1035.

4. Alberti, K. G., Eckel, R. H., Grundy, S. M., Zimmet, P. Z., Cleeman, J. I., Donato, K. A., Smith, S. C., Jr. (2009). Harmonizing the metabolic syndrome: a joint interim statement of the International Diabetes Federation Task Force on Epidemiology and Prevention; National Heart, Lung and Blood Institute; American Heart Association; World Heart Federation; International Atherosclerosis Society; and International Association for the Study of Obesity. Circulation, 120(16), 1640-1645. 
5. American Diabetes Association. (2009). Executive summary: standards of medical care in diabetes--2009. Diabetes Care, 32 Suppl 1, S6-12.

6. Arango, E. F., Pati o, F. A., Quintero, M. A., \& Arenas, M. M. (2011). Levels of physical activity, barriers and stage of change in an urban population from a municipality in Colombia. Colombia M dica, 42(3), 352-361.

7. Asociaci n M dica Mundial. (2008). Declaraci n de Helsinki, 59a Asamblea General. Extra do el 20-06-2010 de http://www.wma.net/ es/30publications/10policies/b3/17c_es.pdf

8. Bergman, P., Grjibovski, A. M., Hagstromer, M., Bauman, A., \& Sjostrom, M. (2008). Adherence to physical activity recommendations and the influence of socio-demographic correlates - a population-based cross-sectional study. BMC Public Health, 8, 367.

9. Burke, L. E., Dunbar-Jacob, J. M., \& Hill, M. N. (1997). Compliance with cardiovascular disease prevention strategies: a review of the research. Ann Behav Med, 19(3), 239-263.

10. Carrieri-Kohlman, V., Nguyen, H. Q., Donesky-Cuenco, D., Demir-Deviren, S., Neuhaus, J., \& Stulbarg, M. S. (2005). Impact of brief or extended exercise training on the benefit of a dyspnea self-management program in COPD. J Cardiopulm Rehabil, 25(5), 275-284.

11. Cupples, M. E., \& McKnight, A. (1994). Randomised controlled trial of health promotion in general practice for patients at high cardiovascular risk. BMJ, 309(6960), 993-996.

12. Chobanian, A. V., Bakris, G. L., Black, H. R., Cushman, W. C., Green, L. A., Izzo, J. L., Jr., Jones, D.W, Materson, J.B., Oparil, S., Wright, J.T. \& Roccella, E. J. (2003). The Seventh Report of the Joint National Committee on Prevention, Detection, Evaluation, and Treatment of High Blood Pressure: the JNC 7 report. JAMA, 289(19), 2560-2572.

13. de Andrade Bastos, A., Salguero, A., Gonzalez-Boto, R., \& Marquez, S. (2006). Motives for participation in physical activity by Brazilian adults. Percept Mot Skills, 102(2), 358-367.

14. Eckel, R. H. (2008). Clinical practice. Nonsurgical management of obesity in adults. N Engl J Med, 358(18), 1941-1950.

15. Expert Panel on Detection, Evaluation, and Treatment of High Blood Cholesterol in Adults (2001). Executive Summary of The Third Report of The National Cholesterol Education Program (NCEP) Expert Panel on Detection, Evaluation, And Treatment of High Blood Cholesterol In Adults (Adult Treatment Panel III). JAMA, 285(19), 2486-2497.

16. Findorff, M. J., Wyman, J. F., \& Gross, C. R. (2009). Predictors of longterm exercise adherence in a community-based sample of older women. J Womens Health (Larchmt), 18(11), 1769-1776.

17. Flegal, K. E., Kishiyama, S., Zajdel, D., Haas, M., \& Oken, B. S. (2007). Adherence to yoga and exercise interventions in a 6-month clinical trial. BMC Complement Altern Med, 7, 37. 
18. Grosbois, J. M., Lamblin, C., Lemaire, B., Chekroud, H., Dernis, J. M., Douay, B., \& Fortin, F. (1999). Long-term benefits of exercise maintenance after outpatient rehabilitation program in patients with chronic obstructive pulmonary disease. J Cardiopulm Rehabil, 19(4), 216-225.

19. Hell n, P., Moreno, J. A., \& Rodr guez, P. L. (2004). Motivos de pr ctica f sico-deportiva en la Regi n de Murcia. Cuadernos de Psicolog a del Deporte, 4, 101-116.

20. Kravitz, R. L., Hays, R. D., Sherbourne, C. D., DiMatteo, M. R., Rogers, W. H., Ordway, L., \& Greenfield, S. (1993). Recall of recommendations and adherence to advice among patients with chronic medical conditions. Arch Intern Med, 153(16), 1869-1878.

21. Lanas, F., Avezum, A., Bautista, L. E., Diaz, R., Luna, M., Islam, S., \& Yusuf, S. (2007). Risk factors for acute myocardial infarction in Latin America: the INTERHEART Latin American study. Circulation, 115(9), 1067-1074.

22. Leijon, M. E., Bendtsen, P., Stahle, A., Ekberg, K., Festin, K., \& Nilsen, P. (2010). Factors associated with patients self-reported adherence to prescribed physical activity in routine primary health care. BMC Fam Pract, 11, 38.

23. Mantilla, S. (2006). Actividad f sica en habitantes de 15 a 49 a os de una localidad de Bogot , Colombia, 2004. Rev Salud P blica 8 (Sup.2), 69-80.

24. Marks, R. (2012). Knee osteoarthritis and exercise adherence: a review. Curr Aging Sci, 5(1), 72-83.

25. Meseguer, C. M., Gal n, I., Herruzo, R., Zorrilla, B., \& Rodriguez-Artalejo, F. (2009). Leisure-time physical activity in a southern European mediterranean country: adherence to recommendations and determining factors. Rev Esp Cardiol, 62(10), 1125-1133.

26. Ministerio de Salud. (1993). Resoluci n N mero 8430 de 1993 (Octubre 4). Extra do el 20-06-2010 de http://www.minsalud.gov.co/Normatividad/RESOLUCION\%208430\%20DE\%201993.pdf

27. Moreno, J. A., Cervell , E., \& Mart nez , A. (2007). Validaci n de la Escala de Medida de los Motivos para la Actividad F sica-Revisada en espa oles: diferencias por motivos de participaci n. Anales de Psico$\log a, 23(1), 167-176$.

28. Netz, Y., Goldsmith, R., Shimony, T., Ben-Moshe, Y., \& Zeev, A. (2011). Adherence to physical activity recommendations in older adults: an Israeli national survey. J Aging Phys Act, 19(1), 30-47.

29. Oldridge, N. B. (1984). Compliance and dropout in cardiac exercise rehabilitation. J Cardiac Rehabil, 4, 166-177.

30. Oldridge, N. B., \& Streiner, D. L. (1990). The health belief model: predicting compliance and dropout in cardiac rehabilitation. Med Sci Sports Exerc, 22(5), 678-683. 
31. Perri, M. G., McAdoo, W. G., McAllister, D. A., Lauer, J. B., \& Yancey, D. Z. (1986). Enhancing the efficacy of behavior therapy for obesity: effects of aerobic exercise and a multicomponent maintenance program. J Consult Clin Psychol, 54(5), 670-675.

32. Robertson, D., \& Keller, C. (1992). Relationships among health beliefs, self-efficacy, and exercise adherence in patients with coronary artery disease. Heart Lung, 21(1), 56-63.

33. Rodr guez, J., Ruiz, F., Pe aloza, E., Eslava, J., G mez, L. C., S nchez, H., ... Botiva, Y. (2009). Encuesta Nacional de Salud 2007. Resultados Nacionales. Bogot : JAVEGRAF.

34. Ryan, R. M., Frederick, C. M., Lepes, D., Rubio, N., \& Sheldon, K. M. (1997). Intrinsic motivation and exercise adherence. International Journal of Sport Psychology, 28, 335-354.

35. S nchez, J. C. (2004). Fitness en las salas de musculaci $n$ (3 $3^{\mathrm{a}}$ ed.). Barcelona: Inde.

36. Schoenborn, C. A., \& Stommel, M. (2011). Adherence to the 2008 adult physical activity guidelines and mortality risk. Am J Prev Med, 40(5), 514-521.

37. Sit, C. H. P., Kerrb, J. H., \& Wong, I. T. F. (2008). Motives for and barriers to physical activity participation in middle-aged Chinese women Psychology of Sport and Exercise, 9(3), 266-283.

38. Steele, B. G., Belza, B., Cain, K. C., Coppersmith, J., Lakshminarayan, S., Howard, J., \& Haselkorn, J. K. (2008). A randomized clinical trial of an activity and exercise adherence intervention in chronic pulmonary disease. Arch Phys Med Rehabil, 89(3), 404-412.

39. Williams, M. H. (2002). Nutrici $n$ para la salud la condici $n f$ sica y el deporte. Barcelona: Paidotribo.

40. Woodard, C. M., \& Berry, M. J. (2001). Enhancing adherence to prescribed exercise: structured behavioral interventions in clinical exercise programs. J Cardiopulm Rehabil, 21(4), 201-209.

Recepción: 05-05-2013

Aprobación: 05-03-2014 